\section{Imaging Software Helps NASA Explore Drug Development}

Rebecca Traino, Strategic Communications Group

There are over 100,000 different proteins in the human body. Each of these proteins plays an important role, such as catalyzing reactions and fighting disease. Researchers at the National Aeronautics and Space Administration (NASA), are looking at the make-up of proteins that are linked to diseases, to lead to the formation of effective drugs to help battle AIDS, diabetes and cancer.

"We are studying the structure of the proteins and how the protein's structure affects its function," says Dr. Russell Judge, a researcher at NASA. "This research will eventually lead to the creation of a drug that interrupts harmful protein activities."

In order to determine the protein structure, scientists have to grow large uniform protein crystals, measure and count the crystals. They must be large and uniform in order for scientists to use $X$-ray diffraction analysis to learn useful structural information. Scientists are performing experiments to find the optimal conditions for protein crystal growth at the biophysics branch of NASA, at Marshall Space Center, in Huntsville, AL. Researchers have found protein crystals grow best in a microgravity environment, which is why NASA has gotten involved. In fact, a recent sample of protein crystals was grown on Senator John Glenn's historic second flight into space. "Protein crystals are made up of thousands of proteins," Judge says. "When they form a crystal they form an ordered array, which gives us a pattern to work back and figure out the position of the atoms inside the molecules."

To find the best conditions for protein crystals growth, the researchers focus on model proteins which are well known and crystallize easily. Lyzolyme, one of the proteins researchers commonly use, is found in chicken egg whites and also in the human body. It has antibacterial properties that fight bacterial cells. Another model protein researchers study is glucose isonerase, which is often used to sweeten soft drinks and converts glucose to fructose.

The crystal formation begins with one of these purified proteins. The proteins are placed in a water solution to which researchers add chemicals that will control the $\mathrm{pH}$ of the solution. This is done to control the growing environment. In order to get the protein to form into crystals, salt is usually added as the precipitation solution, which mixes with the other chemicals within the environment to produce the crystals. Scientists place these proteins on a plastic plate, which has 24 to 96 small

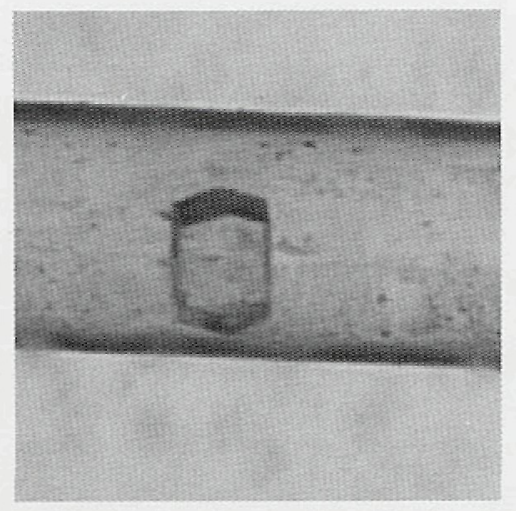

A Lysozyme crystal grown in microgravity and set up in a capillary for $X$-ray analysis wells, and place the plates in incubators at different temperatures for a week.

The samples are retrieved at the end of the week. In that time ten to 20 crystals have grown in each well. The crystals are now ready to be observed under the microscope.

Once the protein crystals are grown to an adequate size, Judge turns them over to a group of scientists who perform an $X$ ray analysis to determine their structure. This describes the structure of the protein on a molecular scale and determines the parts of the protein that are important to its functions.

The process of counting and measuring crystals was traditionally very tedious. After preparing the protein crystal samples, scientists took photographs of the crystals or looked at them directly through the microscopes in order to count the crystals that had grown and to measure their size.

These photographs often took a few days to develop and there was no guarantee that the photographs would develop accurately and provide a usable picture. If the photographs were not taken at the proper angle or were blurry, it would be too late to go back because the experimental conditions had already changed. The work up to that point would be lost.

To alleviate this problem NASA purchased scientific image analysis software. This software allows scientists, researchers, and lab technicians to control the capture of an image seen through a microscope or other imaging device, display that image on the computer screen and manipulate and analyze that image as needed. Scientists at the Marshall Space Flight Center use Image-Pro Plus, from Media Cybernetics (Silver Spring, MD) to capture, analyze and store images of the protein crystals viewed through a microscope. These images allow the researchers to take pictures of several crystal samples at once, and save these images so Judge's research team can analyze them at their convenience.

It also helps the researchers more accurately count the num-

\section{Continued on page 20}

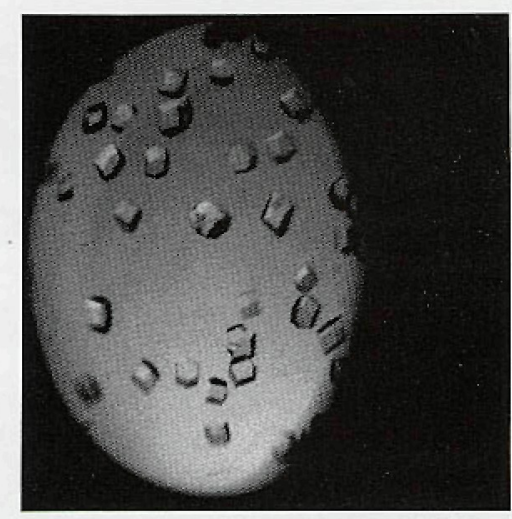

An image of a crystallization plate well, showing a number of lysozyme crystals.

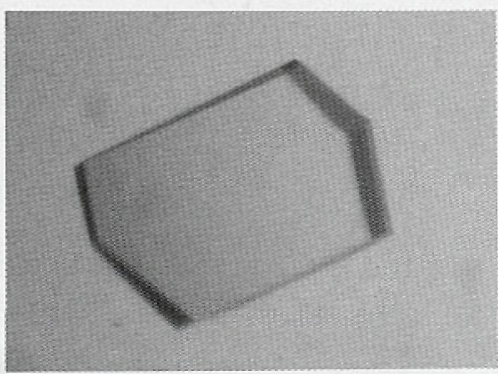

A close up of a lysozyme crystal growth in a crystallization plate. 
also aids in measuring crystal size by using a grid under the microscope to calibrate the measuring function of the software.

"Scientific image analysis software makes our job more convenient," Judge says. "We are able to save images of protein crystals and then have the ability to go back at a later time to measure their shape, size and number. Once we have those numbers we can go back and relate the measurements to the conditions they were grown in, providing the optimal environment to grow large and uniform crystals for later research." These scientific experiments are set up to find the molecular structure of proteins that could lead to the discovery of drugs to prevent and cure diabetes, AIDS, emphysema, cancer and influenza.

Rebecca Traino is a writer with Strategic Communications Group. She can be reached at (301)408-4500 or via email at traino@scgl.com.

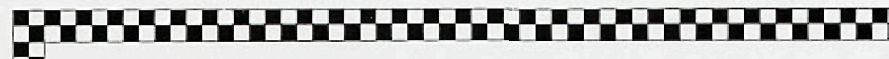 \\ SENIOR LEVEL MICROSCOPIST}

The Department of Microscopy and Microanalysis at Abbott Laboratories is recruiting a senior level microscopist for its Materials Science group.

Requirements include a Ph.D. in Materials Science, Chemistry, or related field, and a working knowledge of most of the following instrumentation: TEM, SEM, EDX, XPS/SIMS, AFM, and light microscopy methods (polarized light, fluorescence, and confocal microscopy). Familiarity with spectroscopic techniques such as FTIR and a strong background in physical and analytical chemistry are desirable. Experience with biological systems would be highly advantageous.

We are looking for a team player with excellent interpersonal skills and the ability to readily adjust to rapidly changing priorities. The successful candidate will seek out and learn new technologies as needed to solve problems related to pharmaceutical and healthcare products. The ability to communicate clearly, both verbally and in writing, is essential.

\section{Essential job functions:}

Independently design and carry out experiments to evaluate mate rials specimens by microscopic and microanalytical techniques.

- Develop methods using multidisciplinary approaches.

- Interpret data and effectively communicate results.

- Review data and reports for scientific integrity and clarity

- Mentor career development of junior scientists.

- Present data at scientific meetings and publish in peer-reviewed journals.

- Provide technical and scientific leadership.

The Department of Microscopy and Microanalysis provides corporatewide support in materials and biological microscopy to all Abbott Laboratories divisions. The facility houses two TEMs (a Philips CM12 STEM and a LEO 910), two SEMs (a Philips XL30 and AMRAY 1830i), three EDX systems, a BioRad MRC 1024 UV confocal scanning laser microscope, a Digital Nanoscope III AFM with Bioscope, a Physical Electronics 5600 XPS/SIMS, and the usual assortment of light microscopes and sample preparatory equipment.

Interested candidates should provide resumes by August 27, 1999 to:

Dr. Jane A. Fagerland

Abbott Laboratories

D45MMAP31

200 Abbott Park Road

Abbott Park, IL 60064-6202

Telephone: (847)935-????

Jane.a.fagerland@abbott.com

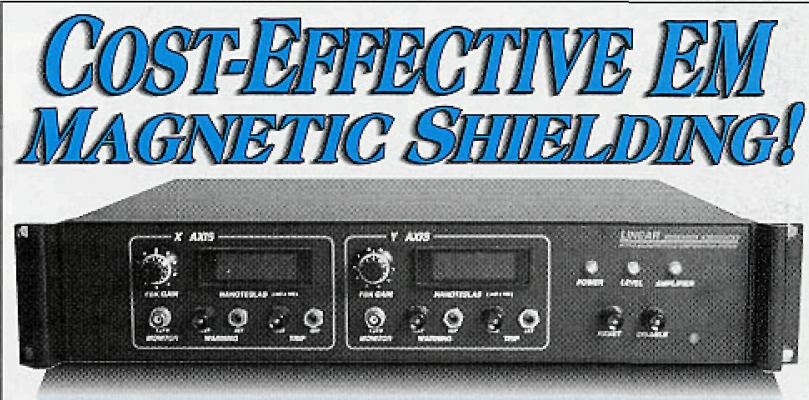

EM sites often require supplemental magnetic shielding to achieve full resolution. LINEAR RESEARCH ASSOCIATES' wideband EMFC-AC electronic active-shielding systems dramatically reduce magnetic fields radiating from nearby a.c. power wiring, ground loops, transformers and related sources. EMFC-QDC extended-range models additionally suppress lowfrequency magnetic disturbances caused by trains, subways, elevators and equipment such as MRI scanners.

Linear Research Associates' EMFC equipment is the highestperformance commercially available magnetic field compensation apparatus in the world. EMFC systems feature state-of-the-art engineeringtt and are U.S.-manufactured to exacting standards. Superior performance and reliability are guaranteed!

Call or fax LRA for complete EMFC-series information. We will also gladly assist with any questions you may have regarding site survey, engineering or EMF issues.

\section{LINEAR RESEARCH ASSOCIATES}

5244 Perry City Road • Trumansburg, NY 14886

Phone (607) 387-3411 • Fax (607) 387-7806

info@linres.com - http://www.linres.com

†† U.S. Patents $5,465,012 ; \quad 5,469,058$

\section{(D) \\ Remove the Veil of Contamination from SEM Samples! NEWI \\ Flash SEM-CLEAN in-situ Cleaning}

- Quickly remove hydrocarbon scum from SEM chamber walls and samples

- Clean samples in-situ

- $\quad$ Fast - less than 5 minutes

- Activated Oxygen cleaning action

- Safe - No sputter etching

- Ideal for Materials Science and Metrology

- No exotic gases - No Argon

- Patent pending

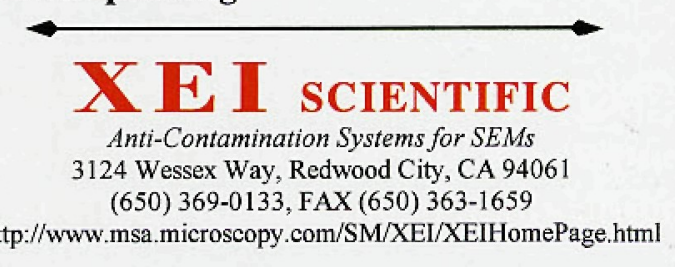

